\title{
Investigation of ancient substructures in the Milky Way: chemical composition study
}

\author{
Edita Stonkutè ${ }^{1}$, Birgitta Nordström ${ }^{2}$, and Gražina Tautvaišiené ${ }^{1}$ \\ ${ }^{1}$ Institute of Theoretical Physics and Astronomy of Vilnius University, \\ Gostauto 12, LT-01108, Vilnius, Lithuania \\ email: edita@itpa.It taut@itpa.It \\ ${ }^{2}$ Niels Bohr Institute, Copenhagen University, Juliane Maries Vej 30, DK-2100, Copenhagen, \\ Denmark, email: birgitta@astro.ku.dk
}

\begin{abstract}
From high resolution spectra taken with the spectrograph FIES on the Nordic Optical Telescope, La Palma, we measure abundances of oxygen and $\alpha$-elements in order to characterize stars which from their dynamical properties are suspected to have originated in disrupted satellites. We find that the chemical composition of investigated stars is homogeneous and distinct from Galactic disk dwarfs, which is providing further evidence of their extragalactic origin.
\end{abstract}

Keywords. Stars: abundances, Galaxy: formation

\section{Introduction}

The formation and evolution of the Milky Way is quite complex and still not fully understood. Helmi et al. (2006) have used a homogeneous data set of about 14.500 F- and G-type stars from the Nordström et al. (2004) catalogue which has complete kinematic, metallicity and age parameters to search for signatures of past accretion in the Milky Way. From correlations between orbital parameters: apocentre (A), pericentre (P) and z-angular momentum $\left(L_{z}\right)$ so called APL space, Helmi et al. identified three new coherent groups of stars and suggested that those might correspond to remains of disrupted satellites. Stars in those groups cluster around regions of roughly constant eccentricity, they have distinct metallicity $[\mathrm{Fe} / \mathrm{H}]$ and age distribution.

Our aim is to measure the detailed elemental abundances of stars belonging to those groups and make a comparison with Galactic disk stars. In this contribution the preliminary chemical composition results are presented for the third stellar group which is of a typical metallicity arround -0.8 dex and a single isochrone age of $\sim 14$ Gyr.

\section{Observations and method of analysis}

The spectra were obtained at the Nordic Optical Telescope with the FIES spectrograph $(R \approx 68000)$ during July of 2008 and reduced with the FIES pipeline FIEStool. The spectra were analysed using a differential model atmosphere technique. The programme packages, developed at Uppsala Astronomical Observatory, were used to carry out the calculations. A set of model atmospheres was taken from the MARCS library (http://marcs.astro.uu.se/).

The effective temperature for the programme stars were taken from Holmberg et al. (2009). We used ionization equilibrium method to find surface gravities of the programme stars by forcing neutral and ionized iron lines to yield the same iron abundances. The microturbulent velocities were determined by forcing Fe I line abundances to be independent of the equivalent width. 


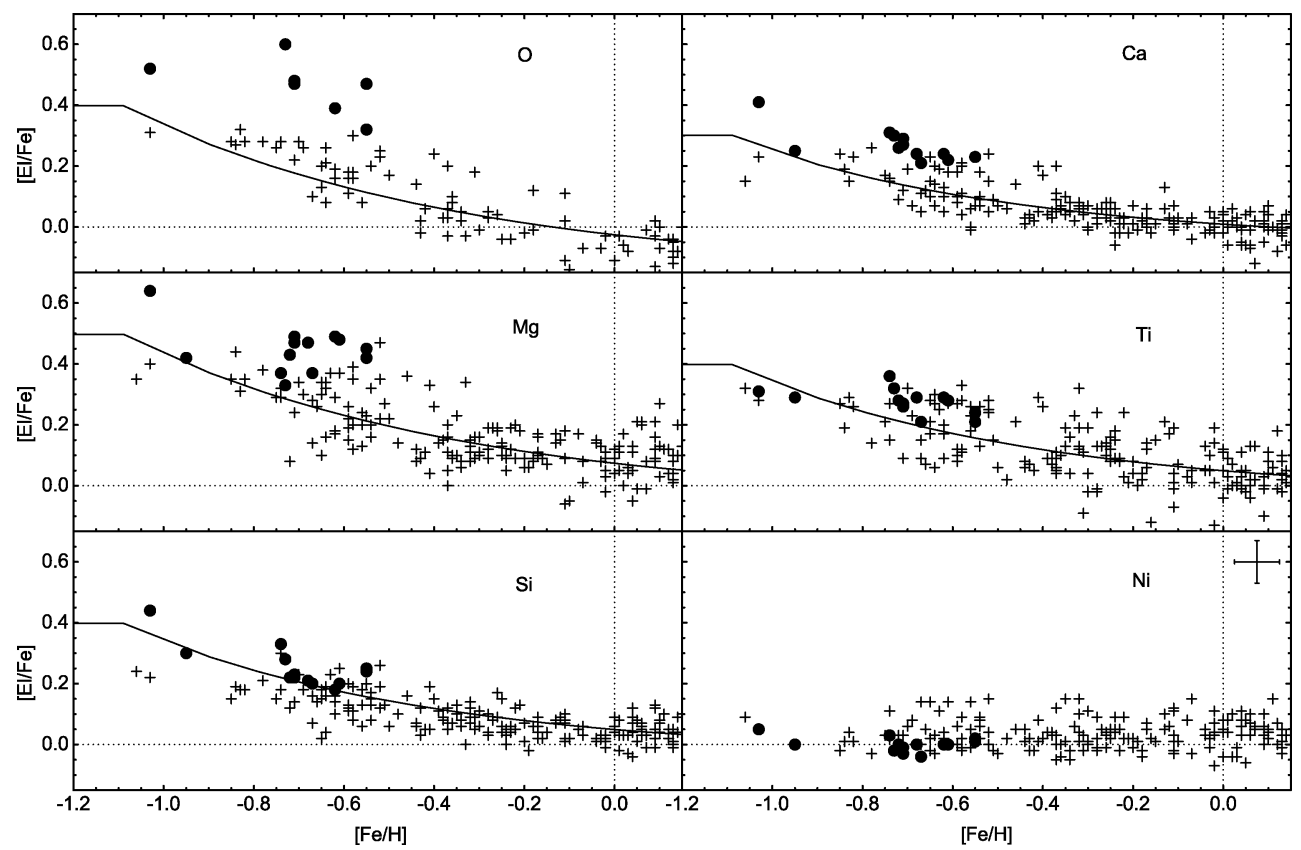

Figure 1. Chemical element abundance ratios in the investigated stars suspected to belong to a disrupted satellite (black dots). The data for the Milky Way disk dwarfs by Edvardsson et al. (1993, plus signs). The Galactic chemical evolution models by Pagel \& Tautvaišienè (1995) are shown by solid lines.

The oxygen abundances were determined from the [O I] line at $6300 \AA$. The atomic data of Ni I were taken from Johansson et al. (2003). The magnesium abundances were determined from the Mg I lines $\lambda 6318.7$ and $6319.2 \AA$ and the non-LTE corrections up to 0.03 dex were calculated and applied using the programme package described by Gratton et al. (1999). More details about the method of analysis can be found in our previous papers (e.g. Tautvaišienè et al. 2005).

\section{Detailed chemical composition}

From the detailed chemical composition of the investigated stars (see Fig. 1) we conclude that the sample of stars is chemically homogeneous, abundances of oxygen and $\alpha$-elements are overabundant in comparison to the Galactic disk dwarfs (Edvardsson et al. 1993) and to the models by Pagel \& Tautvaišienè (1995). These preliminary results provide further evidence that these stars could be of extragalactic origin.

\section{References}

Edvardsson, B., Andersen, J., Gustafsson, B., Lambert, D. L., Nissen, P. E., \& Tomkin, J. 1993, $A \mathscr{E} A, 275,101$

Gratton, R. G., Carretta, E., Eriksson, K., \& Gustafsson, B. 1999, A\&A, 350, 955

Helmi, A., Navarro, J., \& Nordström, B. 2006, MNRAS, Vol. 365, 1309

Holmberg, J., Nordström, B., \& Andersen, B. 2009, A\& $A, 501,941$

Johansson, S., Litzén, U., Lundberg, H., \& Zhang, Z. 2003, ApJ, 584, L107

Nordström, B., Mayor, M., Andersen, J. et al. 2004, A\&\&A 418, 989

Pagel, B. E. J. \& Tautvaišienè, G. 1995, MNRAS, 276, 505

Tautvaišienè, G., Edvardsson, B., Puzeras, E., \& Ilyin, I. 2005, A $\& A$ A, 431, 933 